\section{Commentary: A case study that is more than a typical fascinoma report}

\author{
David D. Yuh, MD, FACS, FACC
}

In their fascinating and beautifully documented case report of a very high left coronary artery take-off, Iacona and colleagues ${ }^{1}$ not only describe an elegantly planned and wellexecuted surgical solution for an extremely rare coronary anomaly, but also put forth alternative strategies to address variations of this anatomic curiosity that they could have encountered. In some respects, the circumstances of this particular case, including older age, tricuspid aortic valve without aortic root dilatation, and sufficient space between the anomalous left coronary artery (LCA) take-off and brachiocephalic artery that permitted conventional distal aortic cannulation and crossclamp placement, simplified the operative strategy. Nevertheless, the critical role of multislice computed tomography and 3-dimensional reconstructions was well highlighted.

Unlike typical case reports, Iacona and colleagues ${ }^{1}$ provide readers the opportunity to think through other interesting, yet plausible scenarios of an anomalous high LCA take-off. For example, if the course of the anomalous LCA took a largely intramural course down the posteromedial aspect of the aneurysmal ascending aorta, would unroofing hamper or preclude adequate reduction aortoplasty? Would unroofing even be necessary with grafting of the left anterior descending and circumflex systems? One can envision an even more challenging scenario of a dilated root in a slightly younger patient without coronary artery disease but with a similarly intramural anomalous LCA whereby unroofing, even at the level of the coronary sinuses, would not be a viable option. In this case, one could consider revascularization of the left anterior descending

From the Department of Surgery, Stamford Hospital, Stamford, Conn.

Disclosures: The author reported no conflicts of interest.

The Journal policy requires editors and reviewers to disclose conflicts of interest and to decline handling or reviewing manuscripts for which they may have a conflict of interest. The editors and reviewers of this article have no conflicts of interest.

Received for publication June 10, 2021; revisions received June 10, 2021; accepted for publication June 18, 2021; available ahead of print June 23, 2021.

Address for reprints: David D. Yuh, MD, FACS, FACC, Department of Surgery, Stamford Hospital, One Hospital Plaza, PO Box 9317, Stamford, CT 06904 (E-mail: DYuh@stamhealth.org).

JTCVS Techniques 2021;8:58-9

2666-2507

Copyright (C) 2021 The Author(s). Published by Elsevier Inc. on behalf of The American Association for Thoracic Surgery. This is an open access article under the CC BY-NC-ND license (http://creativecommons.org/licenses/by-nc-nd/4.0/).

https://doi.org/10.1016/j.xjtc.2021.06.021

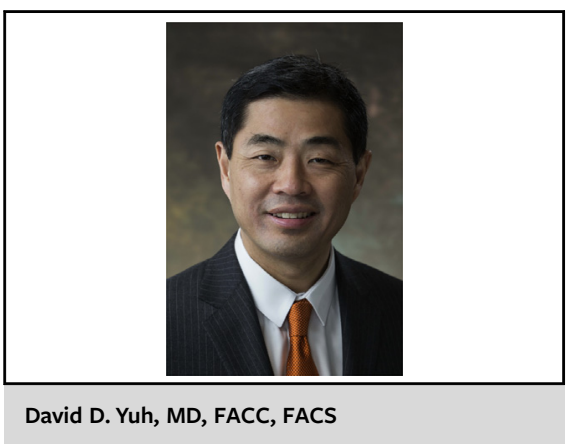

CENTRAL MESSAGE

Cardiac surgeons who treat adults need a working knowledge of the embryologic origins and variations of coronary anomalies to address them while planning otherwise conventional cardiac operations.

and circumflex systems with bilateral internal thoracic arterial grafts as a substitute for LCA reimplantation. Finally, although the authors describe an alternative dual arterial cannulation strategy to address a higher LCA take-off, deep hypothermic circulatory arrest or selective cerebral perfusion approaches might be necessary in the case of a younger patient with ascending aortic aneurysmal disease extending into the proximal aortic arch, permitting mobilization and reimplantation of the LCA into the ascending aortic graft.

Finally, this case report calls attention to the mindbending embryologic origins of the coronary circulation. Although the precise cellular and biochemical interactions leading to normal coronary anatomy have yet to be fully defined, cardiac surgeons who treat adult patients would be well served by familiarizing themselves with what is known about coronary embryology. ${ }^{2,3}$ This would allow them to better appreciate and address the more common coronary anomalies and pathophysiologies they may encounter. Moreover, the importance of recognizing and managing coronary anomalies is indicated by a recent magnetic resonance imaging-based survey suggesting that important coronary anomalies are present in 1,300,000 of the US population and are among the primary causes of sudden cardiac death among athletes and military recruits. ${ }^{4,5}$ I encourage any cardiac surgeon with even a modicum of curiosity as to how the human heart forms to review what has been elucidated. 
Iacona and colleagues ${ }^{1}$ should be congratulated on contributing a case report that does much more than merely document an unusual operative case. The authors take the reader through the process of integrating angiographic, radiographic, and digital images to both clearly illustrate and address an unprecedented case of a fascinating anatomic anomaly.

\section{References}

1. Iacona GM, Pettersson GB, Svensson LG, Bakaeen FG. High take-off of the left coronary artery from the distal ascending aorta. J Thorac Cardiovasc Surg Tech. 2021;8:53-5.
2. Tomanek R, Angelini P. Embryology of coronary arteries and anatomy/pathophysiology of coronary anomalies. A comprehensive update. Int J Cardiol. 2019;28-34.

3. Pérez-Pomares JM, de la Pompa JL, Franco D, Henderson D, Ho S-Y, Houyel L, et al. Congenital coronary artery anomalies: a bridge from embryology to anatomy and pathophysiology - a position statement of the Development, Anatomy, and Pathology ESC Working Group. Cardiovasc Res. 2016; 109:204-16.

4. Angelini P, Cheong BY, Lenge De Rosen VV, Lopez A, Uribe C, Masso AH. Prevalence of high-risk cardiovascular conditions for sport-related sudden death in 5189 schoolchildren studied by magnetic resonance imaging-based screening protocol. Tex Heart Inst J. 2018;45:205-13.

5. Eckart RE, Scoville SL, Campbell CI, Shry EA, Stajduhar KC, Potter RN, et al Sudden death in young adults: a 25 -year review of autopsies in military recruits. Ann Intern Med. 2004;141:829-34. 